Supporting information

\title{
Oral administration of nano-iron sulfide supernatant for the treatment of gallbladder stones with chronic cholecystitis
}

\author{
Liming Ding, Jian Jiang, Lu Cheng, Yanqiu Wang, Wei \\ Zhang, Dandan Li, Zhuobin Xu, Jing Jiang, Zhennan Li*, \\ Lizeng Gao*
}

AUTHOR INFORMATION

Corresponding authors

Lizeng Gao- Institute of Translational Medicine, Medical College, Yangzhou University, Yangzhou 225001, China; CAS Engineering Laboratory for Nanozyme, Institute of Biophysics, Chinese Academy of Sciences, Beijing 100101, China; Email: gaolizeng@ibp.ac.cn. Orcid.org/0000-0003-0265-4027

Zhennan Li- Subei People's Hospital of Jiangsu Province, Yangzhou 225001, China; Email: doclizn@outlook.com

Authors

Liming Ding- Institute of Translational Medicine, Medical College, Yangzhou University, Yangzhou 225001, China 


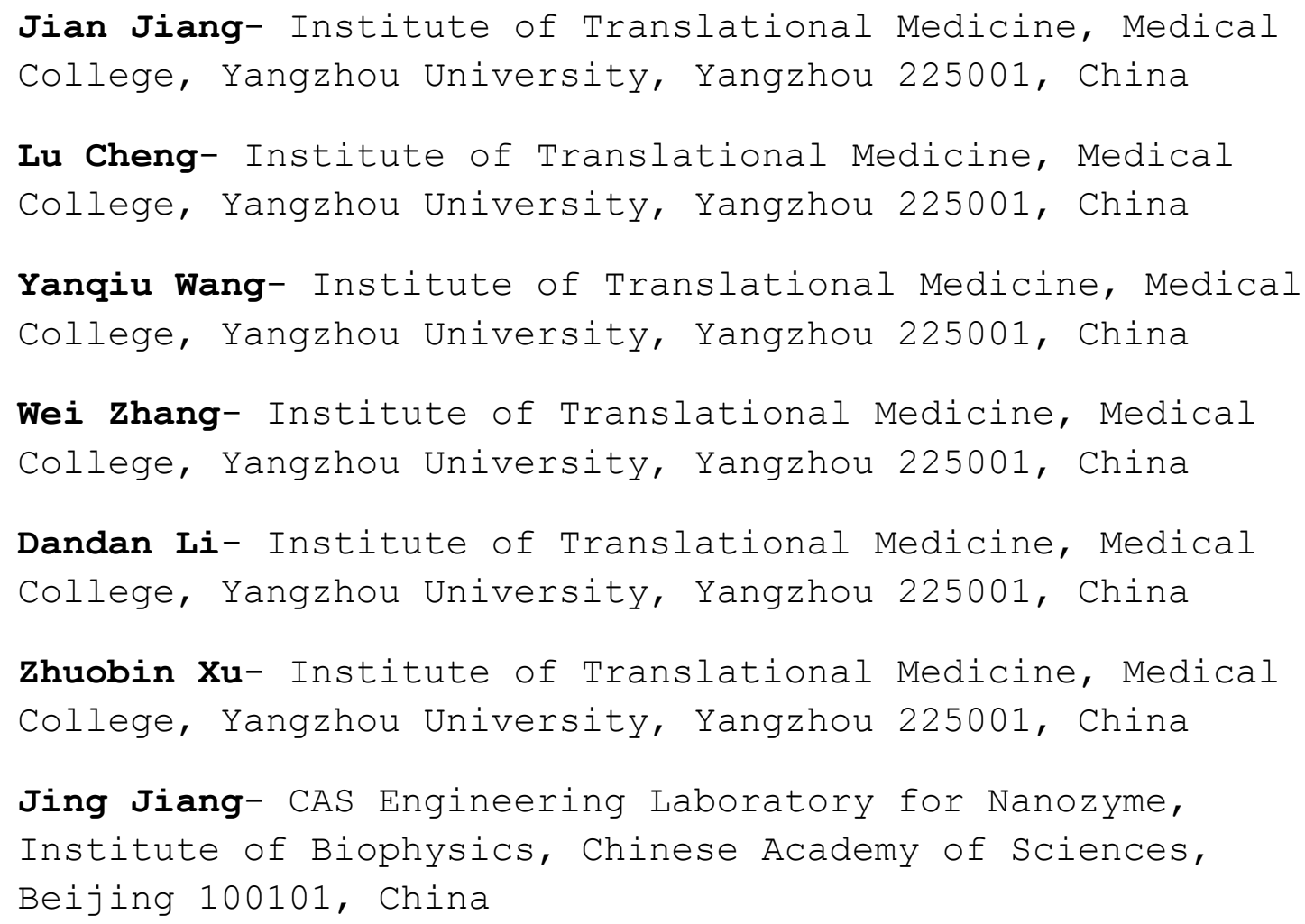

\section{Methods}

\section{Preparation and characterization of iron sulfide its supernate}

Cys-nFeS were prepared using solvothermal reaction. Firstly, $0.82 \mathrm{~g}$ of $\mathrm{FeCl}_{3}$ was dissolved in 40 $\mathrm{mL}$ ethylene glycol and stirred until a clear solution formed. Next, $3.6 \mathrm{~g}$ of $\mathrm{NaOAc}$ and $0.5 \mathrm{~g}$ cysteine (Cys) were added and stirred for 30 min until cysteine was solved completely. The mixture was sonicated for $10 \mathrm{~min}$ and transferred into a Teflonlined stainless-steel reactor. The reactor was sealed and incubated at $200^{\circ} \mathrm{C}$ for $12 \mathrm{~h}$. After that, the mixture was cooled down to room temperature and the precipitate was collected as the products. After washing with ethanol and $\mathrm{H}_{2} \mathrm{O}$ for three times, respectively, the final products were aged at $60^{\circ} \mathrm{C}$ for $3 \mathrm{~h}$. Finally, the prepared Cys-nFeS were stored in a desiccator for the following experiments. The synthesized Cys-nFeS were characterized using scanning electron microscope (SEM, Hitachi S-4800) and transmission electron microscope (TEM, JEOL JEM-1400 $120 \mathrm{kV}$ ), respectively. The supernate of Cys-nFeS was collected by centrifugation at $8000 \mathrm{rpm}$ for $5 \mathrm{~min}$ and the content was analyzed by inductively coupled plasma mass spectrometry after filter with $0.22 \mu \mathrm{m}$ membrane (ICP-MS, Optima $7300 \mathrm{DV}$, PerkinElmer, USA). 
a

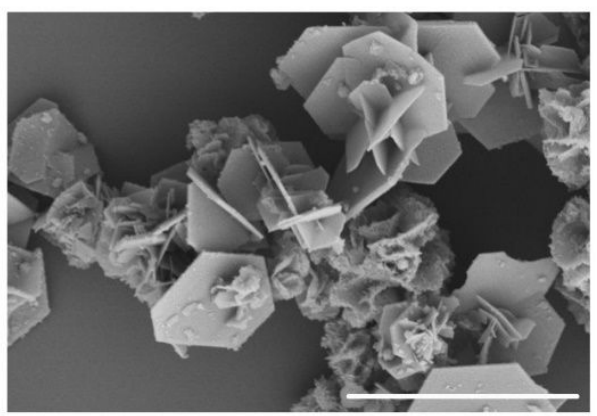

b

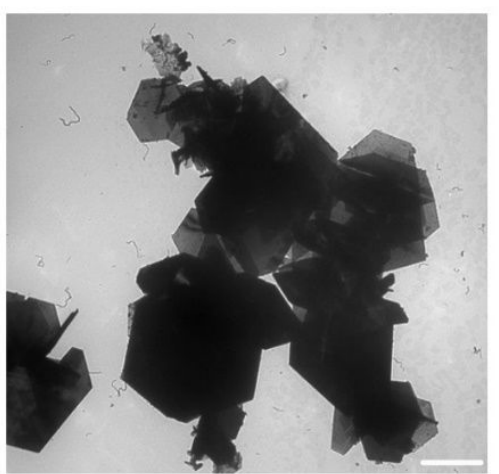

C



Figure S1. Characterization of Cys-nFeS. (a) SEM image of Cys-nFeS. Scale bar: $0.5 \mu \mathrm{m}$. (b) TEM image of Cys-nFeS. Scale bar: $200 \mathrm{~nm}$. (c) The Fe and S content in the supernate of Cys-nFeS dispersion.

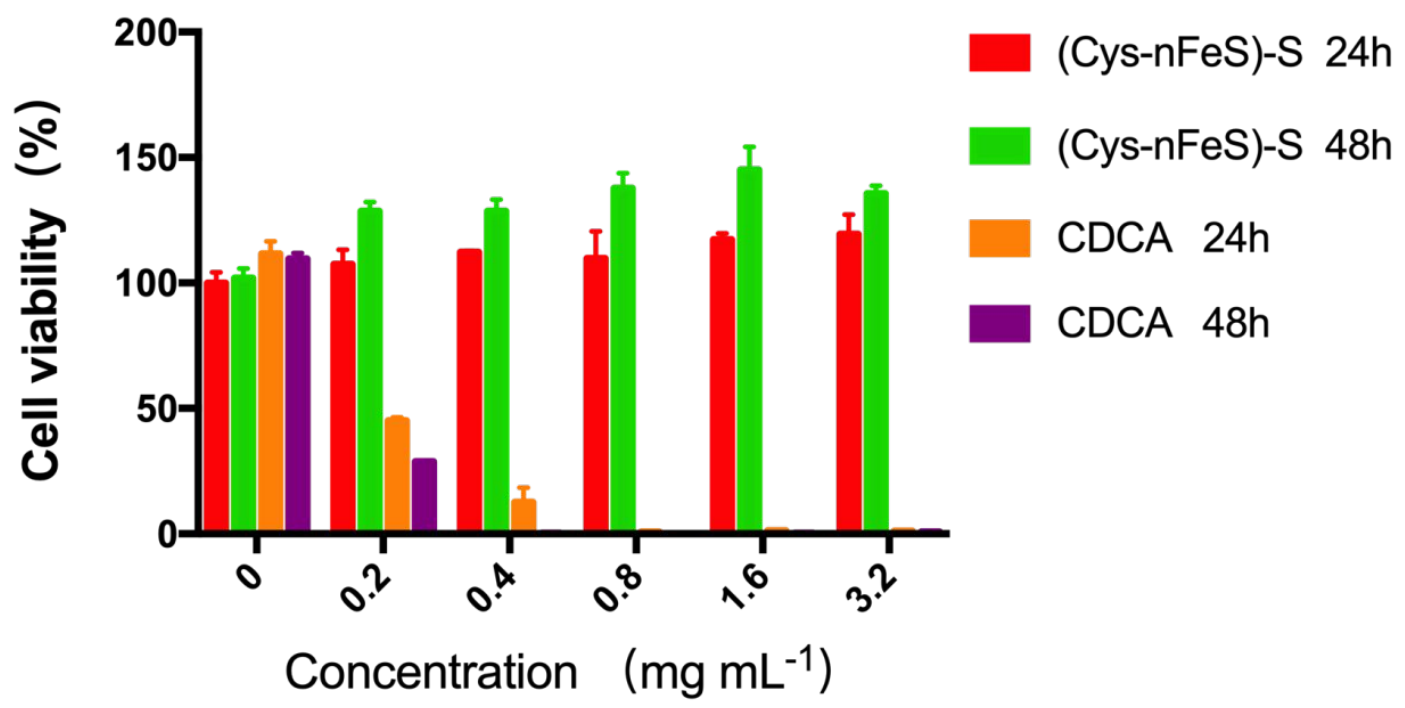

Figure S2. The biocompatibility evaluation of (Cys-nFeS)-S and CDCA by means of L02 cells. Cell viability of L02 cultured with different concentration of (Cys-nFeS)-S and CDCA by means of L02 cells. 
a

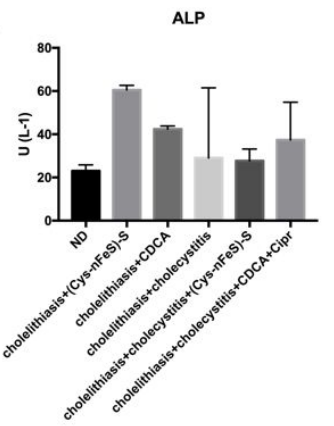

(1)
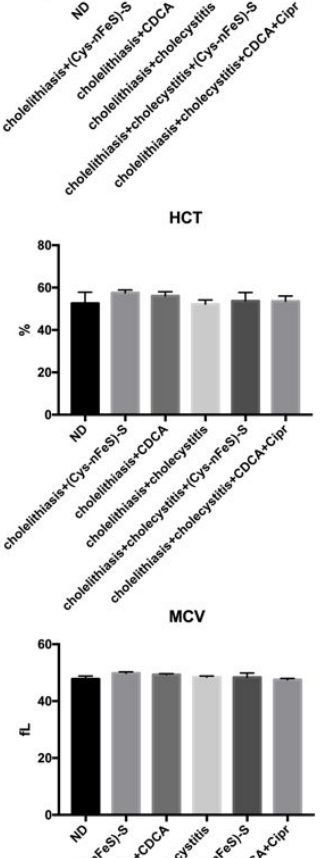


HGB
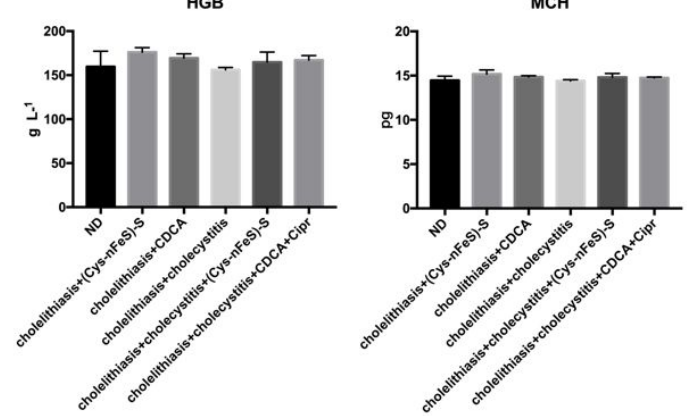
b
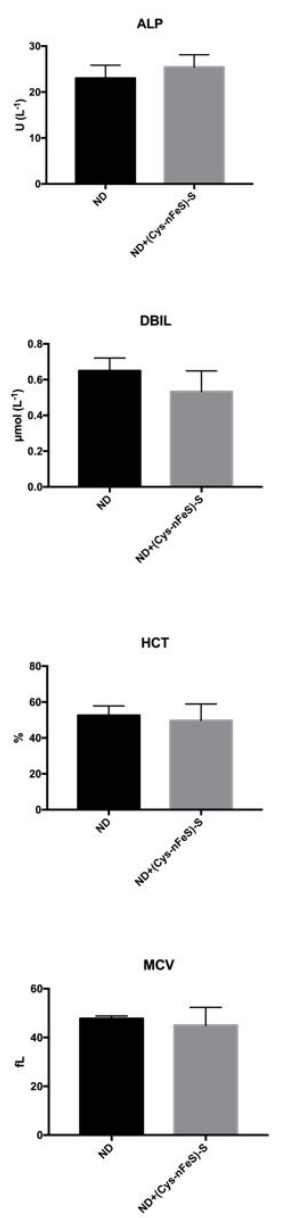
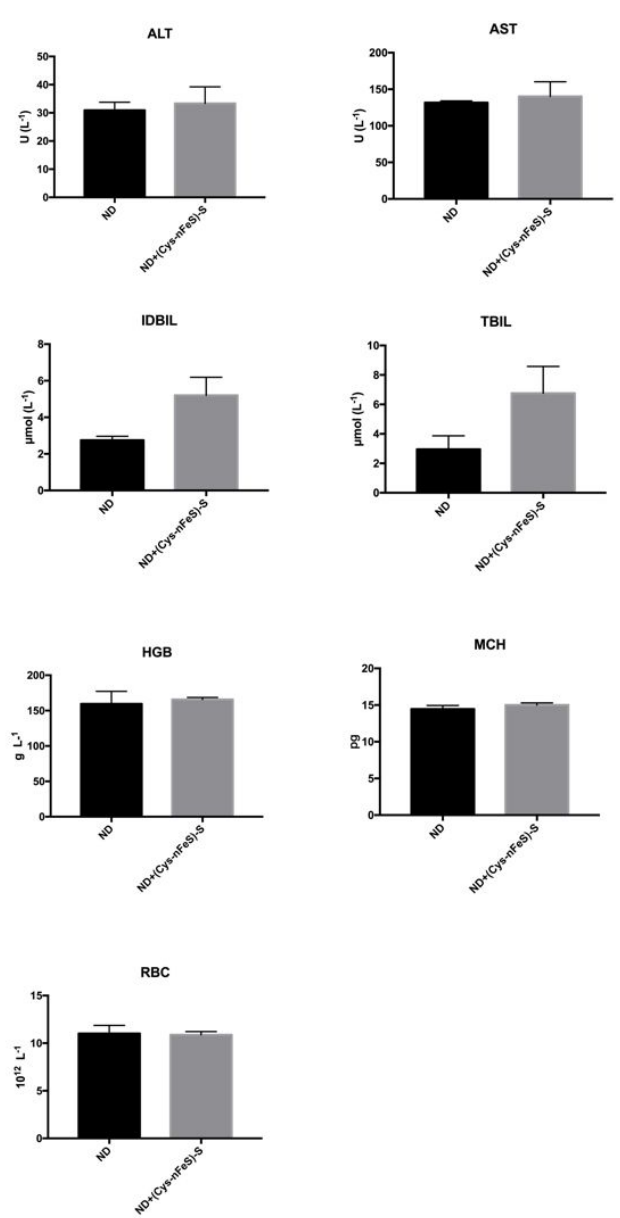

TBIL

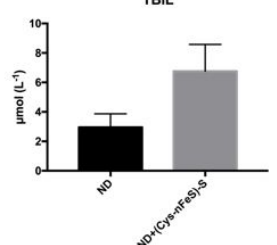

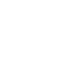




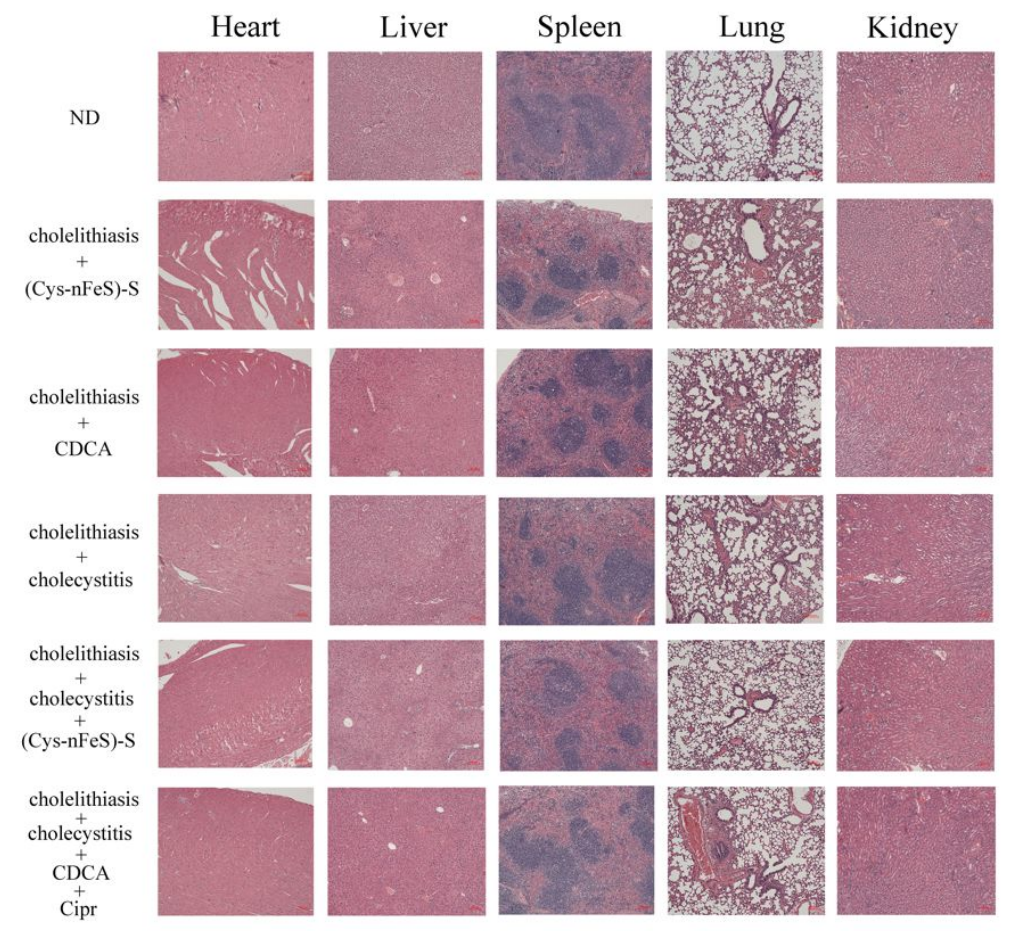

Figure S4. H\&E staining of liver, lung, heart, kidney and spleen after 2 days of different treatment. Scale bars: $100 \mu \mathrm{m}$ 